\title{
ANÁLISE DA UTILIZAÇĀO DE ADITIVOS CRISTALIZANTES PARA AUTORREGENERAÇÃO DO CONCRETO EM TRAÇOS COM DIFERENTE RELAÇÃO ÁGUA/CIMENTO E TIPOS DE CIMÉNTO NACIONAIS
}

\author{
BORJA AGLIARDI, STEPHANY \\ Engenheira Civil \\ UNISINOS \\ São Leopoldo/RS; Brasil \\ stephanyba@edu.unisinos.br \\ DE FRAGA MOREIRA, GIAN \\ Acadêmico de Engenharia Civil \\ UNISINOS/Itt Performance \\ São Leopoldo/RS; Brasil \\ gianfm@unisinos.br
}

PACHECO, FERNANDA

Prof. MSc. Engenheira Civil

UNISINOS/Itt Performance

São Leopoldo/RS; Brasil

fernandapache@unisinos.br

\author{
BATISTELLA, TAUANA \\ Acadêmica de Engenharia de Materiais \\ UNISINOS/Itt Performance \\ São Leopoldo/RS; Brasil \\ tbatistella@edu.unisinos.br
}

\author{
ZAMIS EHRENBRING, HINOEL \\ Prof. MSc. Engenheiro Civil \\ UNISINOS/Itt Performance \\ São Leopoldo/RS; Brasil \\ hzamis@unisinos.br \\ FONSECA TUTIKIAN, BERNARDO \\ Prof. DSc. Engenheira Civil \\ UNISINOS/Itt Performance \\ São Leopoldo/RS; Brasil \\ bftutikian@unisinos.br
}

\section{RESUMO}

Por ser um material durável e possuir elevada resistência à ataques externos e processos de deterioração, o concreto armado é um dos materiais de construção mais utilizados. Todavia, pode apresentar deterioração precoce. Uma das medidas adotadas para ampliar sua vida útil é a incorporação de aditivos cristalizantes, que atuam através da formação de cristais no interior da estrutura do concreto. Além de agir como impermeabilizante, pode ser aplicado para recuperação e reabilitação de estruturas já existentes, pela recuperação das fissuras. Cabe citar que há o surgimento de fissuras, quer seja no estado fresco ou endurecido, e com isso impacta-se a durabilidade do concreto. Este trabalho teve como objetivo realizar um estudo comparativo entre concretos com e sem a incorporação de aditivo cristalizante, com a finalidade de analisar seu comportamento, em relação a resistência característica à compressão. Foram adotadas oito composições, diferenciando-se entre si quanto a adição ou não de aditivo cristalizante, relação água/cimento e variação do tipo de cimento. Os resultados obtidos apontaram que as misturas que receberam a incorporação do cristalizante em sua composição obtiveram um ganho de resistência mais lento nas primeiras idades quando comparadas às amostras sem aditivo. Quanto ao emprego de diferentes tipos de cimentos nas composições, foi possível observar um maior ganho de resistência inicial nas misturas com cimento CP II F, sendo obtidos resultados menores de resistência nas amostras com cimento CP IV.

Palavras-chave: Concreto, aditivo cristalizante, fissuras, durabilidade.

\section{ABSTRACT}

Because a durable material has high resistance to external attack and deterioration processes, or reinforced concrete is one of the most widely used building materials. However, it may present early deterioration. One of the measures taken to extend its service life is to incorporate crystallizing additives, which act by forming crystals within the concrete structure. In addition to acting as a waterproofing agent, it can be applied for restoration of existing structures. Given to the expressive emergence of fissures, whether a fresh or hardened state, and the consequence in terms of damage from their appearance, this work aimed to carry out a comparative study between concretes with and without incorporation of crystallizing additive, with an analysis test of its own. This work aim objective to comparative study between concretes with and without the incorporetion of crystallizing additive in order to analyze its behavior, in relation to the characteristic compressive strength. Eight compositons were adopted, differing from each other the addition or not of crystallizing additive, water/cement and cement type variation. The obtained results pointed that mixtures received the incorporation of crystallizer in its composition had a slow resistance gain in the firts ages when compared to the samples without additive. About the use of diferente types of cement in the composition, it was possible to observe a higher initial strength gain in CP II F cement traces, and lower resistence results were obtained in CP IV cement samples.

Keywords: concrete, crystallinzing additive, fissures, wear. 


\section{INTRODUÇÃO}

O concreto é um dos materiais mais utilizados na construção civil atualmente, mesmo com o crescimento do uso de materiais como o aço e a madeira. Dentre os motivos para elevada empregabilidade, tem-se sua durabilidade. Mesmo sendo um material durável e resistente, ao longo dos anos houve um crescimento nas manifestações patológicas observadas em estruturas de concreto armado (MEHTA e MONTEIRO, 2014).

Um dos fenômenos com maior frequência de ocorrência em elementos de concreto, tanto no estado fresco quanto no endurecido, é a fissuração. Sua ocorrência pode estar relacionada a diferentes causas, como retração, movimentações térmicas, aplicação de cargas e contato com agentes deteriorantes. A abertura das fissuras age como mecanismo de entrada de agentes nocivos ao concreto. Uma vez em contato com a estrutura, os agentes deteriorantes contribuem para a redução da durabilidade do concreto (NEVILLE, 2013). Kaur et al (2019) afirmam que as fissuras, tão logo surgem nos elementos de concreto devem ser tratadas, de modo que não haja tempo até que os agentes deteriorantes penetrem na armadura, causando outros danos patológicos e sua deterioração. Cabe destacar que estudos já apontam a rápida atuação dos aditivos cristalizantes nas fissuras ocorridas nas idades iniciais do concreto.

Conforme Ferrara et al. (2018), a utilização de concretos autorregenerantes ou autocicatrizantes prolonga a vida útil da estrutura e contribui para o meio ambiente. Segundo os autores, a garantia de durabilidade e aumento da vida útil é possível devido ao controle e reparo do concreto nas fases iniciais de fissuração, impossibilitando a entrada de meios agressivos para o interior da estrutura. Outro destaque importante relacionado aos concretos com capacidade de autocura é a redução da necessidade de manutenções e reparos (AHN e KISHI, 2010). Existem diferentes mecanismos para promoção da autorregeneração, estudados por diferentes linhas de pesquisas (TAKAGI; LIMA; HELENE, 2012). Um dos mecanismos existentes para a autocicatrização do concreto é a incorporação de agentes cicatrizantes na mistura (WIKTOR e JONKERS, 2011).

$\mathrm{O}$ aditivo cristalizante reage com a água e com os produtos do processo de hidratação, resultando em uma reação química que causa o preenchimento dos vazios e fissuras presentes no concreto com os cristais. Segundo Ourives e Bilesky (2009), o cristalizante é resistente à ação da intempérie e de águas agressivas, uma vez em contato com a água, os cristais se desenvolvem não permitindo a sua passagem, tornando o concreto menos permeável. Caso haja o surgimento de novas fissuras ao longo da vida útil, os cristais se desenvolvem e impedem a percolação de água.

No caso da autorregeneração, é possível a recuperação da resistência original do concreto, enquanto na autocicatrização as fissuras são seladas sem recuperação das propriedades mecânicas do elemento estrutural (VAN BREUGEL, 2007). A autorregeneração proporcionada pelos adititivos é considerada autônoma, pois emprega materiais visando em específico proporcionar sua capacidade de autocicatrização. Aplicado sobre o concreto, o tratamento é capaz de gerar formações cristalinas profundas, tornando-se parte integrante do mesmo e formando uma barreira definitiva que sela os poros e capilaridades, impedindo a penetração da água, mesmo sob altas pressões hidrostáticas. A reação cristalina se propaga dentro da matriz do concreto como um resultado de três fatores individuais ou combinações: osmose, movimento Browniano e reações de partículas secas. A profundidade da penetração e o tempo envolvido para a cristalização, dependem primeiramente da presença de umidade e dos ingredientes do concreto, além das propriedades físicas do concreto como consumo de cimento, densidade e compactação. Concretos mais porosos possuem maiores velocidades de penetração e nos concretos de menor porosidade a atuação se concentra mais nas microfissurações. (TAKAGI; LIMA; HELENE, 2012).

Considerando o cenário apresentado, este estudo avaliou o desenvolvimento de concretos autorregenerantes autônomos, com uso de aditivos cristalizantes. Foram consideradas amostras com uso de diferentes aglomerantes, com cimento CPII F e CP IV. Para cada um dos cimentos variou-se ainda a relação a/c e o uso ou não do aditivo cristalizante. As misturas foram avaliados em relação a sua resistência à compressão e a eficácia da incorporação do aditivo cristalizante.

\section{PROGRAMA EXPERIMENTAL}

Primeiramente definiu-se os traços (misturas) de concreto. Nos traços de igual relação a/c foram dosados com a mesma composição, variando apenas o tipo de cimento e a presença ou não de aditivo cristalizante. No total foram feitos oito traços de concreto, variando a sua composição quanto à relação a/c e tipo de aglomerante, assim como, a incorporação ou não de aditivo cristalizante. Apresentam cimento do tipo CP II F40 ou CPIV na sua composição, com relações a/c de 0,45 e 0,70 respectivamente e com e sem aditivo cristalizante. Então, os traços foram dosados e moldados 14 amostras para cada traço de concreto, após $24 \mathrm{~h}$ da moldagem, os corpos de prova foram desmoldados e colocados em cura úmida 
conforme a NBR 5738 (ABNT, 2015) e NBR 9479 (ABNT, 2006). Quando no estado endurecido foram realizados os ensaios de resistência mecânica aos 7, 14, 28 e 56 dias de idade, conforme a NBR 5739 (ABNT, 2018). O programa experimental deste estudo foi desenvolvido em três etapas, denominadas como Etapa I, Etapa II e Etapa III. Os processos desenvolvidos em cada etapa estão apresentados no fluxograma da Figura 1.

ETAPA 1

Figura 1 - Etapas do estudo experimental

ETAPA 2

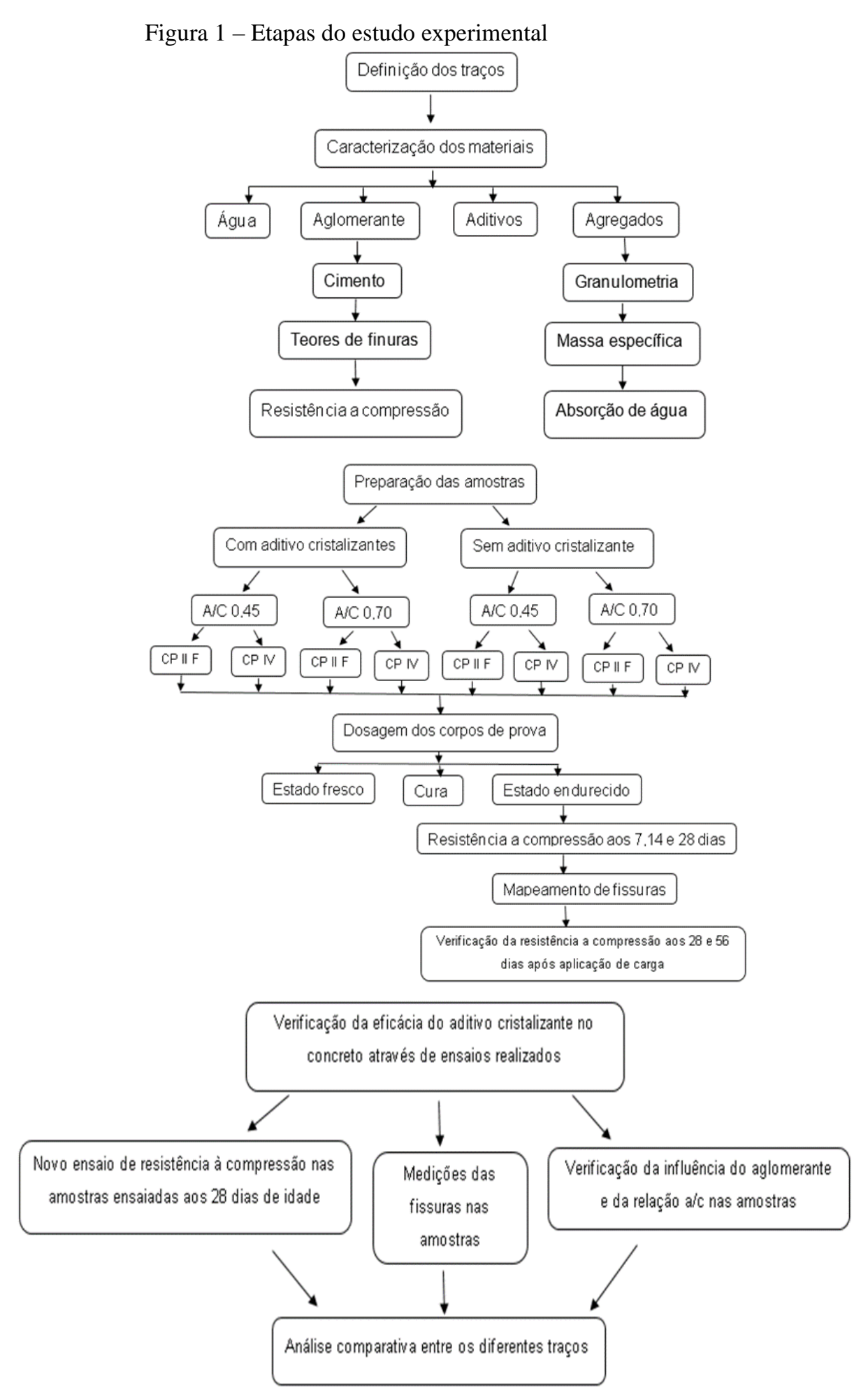

ETAPA 3 


\subsection{Materiais}

Foram utilizados dois tipos de cimento nas misturas, CP II F (Cimento Portland com adição de fíler) e CP IV (Cimento Portland Pozolânico). Para este estudo foram realizadas as caracterizações físicas de ambos os agregados - miúdo e graúdo, e os tipos de brita nomeados comercialmente de brita 0 e brita 1. Foram utilizados dois aditivos nas dosagens dos concretos para realização da análise comparativa, sendo eles: aditivo polifuncional de linha e aditivo cristalizante. A dosagem de aditivo funcional adotada foi de $0,8 \%$ sobre o peso de cimento das composições em cada uma das amostras.

\subsubsection{Caracterização dos agregados}

A norma adotada como referência para os ensaios de granulometria foi a NBR NM 248 (ABNT, 2003). A Tabela 1 apresenta os resultados da composição granulométrica, módulo de finura e dimensão máxima dos agregados miúdos, bem como a Tabela 2 apresenta as mesmas informações para os agregados graúdos.

Tabela 1 - Caracterização física dos agregados miúdos

\begin{tabular}{ccc}
\hline Massa específica (NBR NM 52:2009) & Areia Fina & Areia Média \\
\hline Agregado seco $\left(\mathrm{g} / \mathrm{cm}^{3}\right)$ & 2,67 & 2,66 \\
\hline Saturada superfície seca $\left(\mathrm{g} / \mathrm{cm}^{3}\right)$ & 2,69 & 2,69 \\
\hline Massa específica $\left(\mathrm{g} / \mathrm{cm}^{3}\right)$ & 2,71 & 2,72 \\
\hline Análise granulométrica $(\mathbf{N B R}$ NM 248:2003) & & \\
\hline Dimensão máxima característica & 0,6 & 4,8 \\
\hline Módulo de finura & 0,97 & 2,39 \\
\hline
\end{tabular}

Tabela 1 - Caracterização física dos agregados graúdos

\begin{tabular}{ccc}
\hline Massa específica (NBR NM 53:2009) & Brita 0 & Brita 1 \\
\hline Agregado seco $\left(\mathrm{g} / \mathrm{cm}^{3}\right)$ & 2,9 & 2,93 \\
\hline Saturado superfície seca $\left(\mathrm{g} / \mathrm{cm}^{3}\right)$ & 2,94 & 2,93 \\
\hline Massa específica $\left(\mathrm{g} / \mathrm{cm}^{3}\right)$ & 3 & 2,95 \\
\hline Análise granulométrica $(\mathbf{N B R}$ NM 248:2003) & & \\
\hline Dimensão máxima característica & 12,5 & 25,4 \\
\hline Módulo de finura & 6,24 & 7,02 \\
\hline
\end{tabular}

\subsection{Composição dos traços}

Para o estudo foram realizados oito traços de concreto, variando a sua composição quanto à relação a/c e tipo de aglomerante, assim como, a incorporação ou não de aditivo cristalizante. A Tabela 3 apresenta a identificação por códigos e relação das composições de concreto estudadas. Aplicou-se a numeração do tipo de cimento, seguida do valor da relação a/c utilizada. Por fim, a nomenclatura que contém CR é atribuída aos traços que contém aditivo cristalizante.

Tabela 3- Códigos e nomenclaturas dos concretos

\begin{tabular}{ll}
\hline Código & \multicolumn{1}{c}{ Nomenclatura } \\
\hline II.0,45(1).CR & Concreto ac 0,45 CP II F40 com aditivo cristalizante \\
\hline II.0,45(2) & Concreto ac 0,45 CP II F40 sem aditivo cristalizante \\
\hline II.0,70(3).CR & Concreto ac 0,70 CP II F40 com aditivo cristalizante \\
\hline II.0,70(4) & Concreto ac 0,70 CP II F40 sem aditivo cristalizante \\
\hline IV.0,45(5).CR & Concreto ac 0,45 CP IV com aditivo cristalizante \\
\hline IV.0,45(6) & Concreto ac 0,45 CP IV sem aditivo cristalizante \\
\hline IV.0,70(7).CR & Concreto ac 0,70 CP IV com aditivo cristalizante \\
\hline IV.0,70(8) & Concreto ac 0,70 CP IV sem aditivo cristalizante \\
\hline
\end{tabular}


Nas Tabelas 4 e 5 são apresentados as misturas que apresentam cimento do tipo CP II F40 na sua composição, com relações a/c de 0,45 e 0,70 respectivamente. Foram realizados quatro traços para cada tipo de cimento, variando a relação a/c e a presença ou não de aditivo cristalizante. As composições dos concretos que não recebem a incorporação de aditivo cristalizante na mistura é a mesma, diferindo apenas quanto à sua presença ou não. As composições com cimento do tipo CP IV, com e sem aditivo cristalizante e com a/c 0,45 e 0,70, são apresentadas nas Tabelas 6 e 7 . Todas as composições apresentadas são referentes a um volume de $1 \mathrm{~m}^{3}$. O volume dosado por traço para ensaios no estado fresco e moldagem das amostras foi de $30 \mathrm{~L}$, desta forma a composição foi ajustada para o volume realizado.

Tabela 4- Composição dos traços de concreto com cimento CP II F40 e ac 0,45

\begin{tabular}{lccc}
\hline \multicolumn{2}{c}{ Composição dos traços de concretos com CP II F40 - ac 0,45 } \\
\hline \multirow{2}{*}{ Composição } & Unid. & \multicolumn{3}{c}{ Traço } \\
\cline { 2 - 4 } & & 1 & 2 \\
\hline Água & $\mathrm{L}$ & 176 & 176 \\
\hline Aditivo cristalizante & $\mathrm{Kg}$ & 3,129 & - \\
\hline Areia fina & $\mathrm{Kg}$ & 228 & 228 \\
\hline Areia média & $\mathrm{Kg}$ & 532 & 532 \\
\hline Brita 0 & $\mathrm{Kg}$ & 229 & 229 \\
\hline Brita 1 & $\mathrm{Kg}$ & 921 & 921 \\
\hline Aditivo de linha & $\mathrm{Kg}$ & 3,129 & 3,129 \\
\hline Cimento CP II F 40 & $\mathrm{Kg}$ & 391 & 391 \\
\hline
\end{tabular}

Tabela 5- Composição dos traços de concreto com cimento CP II F40 e ac 0,70

\begin{tabular}{|c|c|c|c|}
\hline \multicolumn{4}{|c|}{ Composição dos traços de concretos com CP II F40 - ac 0,70 } \\
\hline \multirow{2}{*}{ Composição } & \multirow{2}{*}{ Unid. } & \multicolumn{2}{|c|}{ Traço } \\
\hline & & 3 & 4 \\
\hline Água & $\mathrm{L}$ & 176 & 176 \\
\hline Aditivo cristalizante & $\mathrm{Kg}$ & 2,01 & - \\
\hline Areia fina & $\mathrm{Kg}$ & 264 & 264 \\
\hline Areia média & $\mathrm{Kg}$ & 617 & 617 \\
\hline Brita 0 & $\mathrm{Kg}$ & 229 & 229 \\
\hline Brita 1 & $\mathrm{Kg}$ & 921 & 921 \\
\hline Aditivo de linha & $\mathrm{Kg}$ & 2,01 & 2,01 \\
\hline Cimento CP II F 40 & $\mathrm{Kg}$ & 251 & 251 \\
\hline
\end{tabular}

Tabela 6- Composição dos traços de concreto com cimento CP IV e ac 0,45

\begin{tabular}{|c|c|c|c|}
\hline \multicolumn{4}{|c|}{ Composição dos traços de concretos com CP IV - ac 0,45} \\
\hline \multirow{2}{*}{ Composição } & \multirow{2}{*}{ Unid. } & \multicolumn{2}{|c|}{ Traço } \\
\hline & & 5 & 6 \\
\hline Água & $\mathrm{L}$ & 176 & 176 \\
\hline Aditivo cristalizante & $\mathrm{Kg}$ & 3,129 & - \\
\hline Areia fina & $\mathrm{Kg}$ & 228 & 228 \\
\hline Areia média & $\mathrm{Kg}$ & 532 & 532 \\
\hline Brita 0 & $\mathrm{Kg}$ & 229 & 229 \\
\hline Brita 1 & $\mathrm{Kg}$ & 921 & 921 \\
\hline Aditivo de linha & $\mathrm{Kg}$ & 3,129 & 3,129 \\
\hline Cimento CP IV & $\mathrm{Kg}$ & 391 & 391 \\
\hline
\end{tabular}

Tabela 7- Composição dos traços de concreto com cimento CP IV e ac 0,70 


\begin{tabular}{|c|c|c|c|}
\hline \multicolumn{4}{|c|}{ Composição dos traços de concretos com CP IV - ac 0,70 } \\
\hline \multirow{2}{*}{ Composição } & \multirow{2}{*}{ Unid. } & \multicolumn{2}{|c|}{ Traço } \\
\hline & & 7 & 8 \\
\hline Água & $\mathrm{L}$ & 176 & 176 \\
\hline Aditivo cristalizante & $\mathrm{Kg}$ & 2,01 & - \\
\hline Areia fina & $\mathrm{Kg}$ & 264 & 264 \\
\hline Areia média & $\mathrm{Kg}$ & 617 & 617 \\
\hline Brita 0 & $\mathrm{Kg}$ & 229 & 229 \\
\hline Brita 1 & $\mathrm{Kg}$ & 921 & 921 \\
\hline Aditivo de linha & $\mathrm{Kg}$ & 2,01 & 2,01 \\
\hline Cimento CP IV & $\mathrm{Kg}$ & 251 & 251 \\
\hline
\end{tabular}

\subsection{Ensaios realizados}

\subsubsection{Fissuração}

Após a confecção das misturas, foram acrescentados no concreto em seu estado fresco, pequenas placas acrílicas nas amostras, onde essas possuem dimensão de $2 \mathrm{~cm}$ de profundidade, $4 \mathrm{~cm}$ de largura e $0,3 \mathrm{~cm}$ de espessura. A profundidade de $2 \mathrm{~cm}$ foi adotada de forma a possibilitar a análise em toda extensão da fissura e a verificação da presença de possível desenvolvimento de material regenerador em seu interior. A espessura da fissura de $0,3 \mathrm{~cm}$ foi considerada assim pois buscou-se avaliar a limitação apresentada em bibliografia no que tange a espessura máxima cicatrizável. A Imagem 1 apresenta o corpo de prova com a fissura induzida.

\section{Imagem 1 - Fissura induzida no corpo de prova}
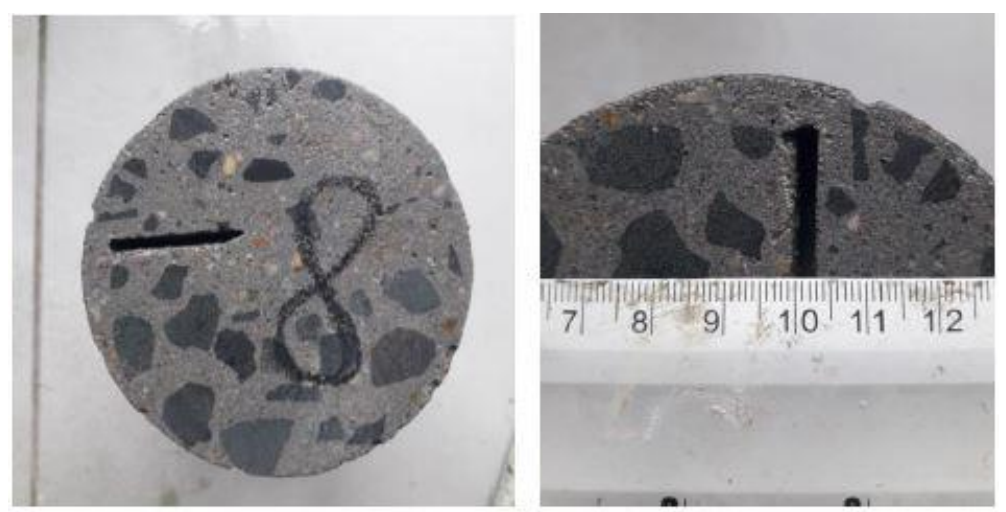

\subsubsection{Ensaios de compressão}

A resistência à compressão do concreto foi verificada de acordo com o procedimento de ensaio da norma NBR 5739 (ABNT, 2018). Foram utilizados para o ensaio amostras moldadas em corpos de prova cilíndricos de $20 \mathrm{~cm}$ de altura e $10 \mathrm{~cm}$ de largura. As amostras foram mantidas em cura submersa até a data de realização dos ensaios de rompimento aos 7, 14, 28 e 56 dias de idade, com e sem aditivo cristalizante.

\section{RESULTADOS E DISCUSSÕES}

\subsection{Mapeamento das fissuras}

Na Tabela 8 é apresentado o mapeamento das fissuras aos 7, 14, 28 e 56 dias de idade, contendo a espessura de cada amostra no momento prévio ao ensaio de compressão axial. 
Tabela 8 - Mapeamento das fissuras

\begin{tabular}{|c|c|c|c|c|}
\hline \multirow[b]{2}{*}{ Traço } & \multicolumn{4}{|c|}{ Abertura de fissuras (mm) } \\
\hline & 07 dias & 14 dias & 28 dias & 56 dias \\
\hline II.O,45(1).CR & $3 \mathrm{~mm}$ & $3 \mathrm{~mm}$ & $3 \mathrm{~mm}$ & $3 \mathrm{~mm}$ \\
\hline II.O,45(2) & $3 \mathrm{~mm}$ & $3 \mathrm{~mm}$ & $3 \mathrm{~mm}$ & $3 \mathrm{~mm}$ \\
\hline II.O,70(3).CR & $3 \mathrm{~mm}$ & $3 \mathrm{~mm}$ & $3 \mathrm{~mm}$ & $3 \mathrm{~mm}$ \\
\hline II.O,70(4) & $3 \mathrm{~mm}$ & $3 \mathrm{~mm}$ & $3 \mathrm{~mm}$ & $3 \mathrm{~mm}$ \\
\hline IV.O,45(5).CR & $3 \mathrm{~mm}$ & $3 \mathrm{~mm}$ & $3 \mathrm{~mm}$ & $3 \mathrm{~mm}$ \\
\hline IV.0,45(6) & $3 \mathrm{~mm}$ & $3 \mathrm{~mm}$ & $3 \mathrm{~mm}$ & $3 \mathrm{~mm}$ \\
\hline IV.O,70(7).CR & $3 \mathrm{~mm}$ & $3 \mathrm{~mm}$ & $3 \mathrm{~mm}$ & $3 \mathrm{~mm}$ \\
\hline IV.0,70(8) & $3 \mathrm{~mm}$ & $3 \mathrm{~mm}$ & $3 \mathrm{~mm}$ & $3 \mathrm{~mm}$ \\
\hline
\end{tabular}

As fissuras provocadas nas amostras mantiveram as suas aberturas de 3mm nas idades de 7, 14,28 e 56 dias, não podendo ser avaliada a efetividade do aditivo cristalizante quanto à autorregeneração do concreto, através do fechamento das fissuras. O fabricante do aditivo cristalizante indica que a abertura máxima de fissuras onde é comprovada a eficácia do aditivo é de 0,4mm, assim como outros estudos já realizados com esse tipo de autorregenerante e com outros métodos possíveis. Conforme Mori, Kuramoto, Takagi, Horie, \& Tanimoto (1996), a incorporação de impermeabilizante como o catalisador cristalino pode provocar uma impermeabilização por cristalização da estrutura, desde que as fissuras possuam uma abertura máxima de 0,4mm. Todavia, nesse estudo, não percebeu-se nem a formação de produtos na parede da fissura, um fenômeno já relatado na bibliografia.

Em outro estudo realizado por Sisomphon, Copuroglu e Koenders (2012), que teve como objetivo verificar o potencial de autocura em argamassas autocicatrizantes com incorporação de aditivo expansivo e aditivo cristalizante, verificou-se o fechamento das fissuras provocadas com espessuras entre 0,25 e $0,4 \mathrm{~mm}$. Por possuírem uma abertura superior ao indicado pelo fabricante como limite máximo de atuação do aditivo cristalizante, de forma a verificar a eficácia do produto em situações reais do aparecimento de fissuras em elementos estruturais, as fissuras provocadas de $3 \mathrm{~mm}$ nas amostras não apresentaram um completo fechamento, o que comprovaria a eficácia do aditivo na autorregeneração do concreto. Porém, com esta abertura foi possível verificar se houve ou não o desenvolvimento de material regenerador no interior das fissuras, assim como, sinais de cristalização nestes pontos.

Fissuras com a espessura das provocadas neste estudo, apesar de não enquadrarem-se nas recomendações de aplicação do fabricante para aberturas de no máximo 0,4mm, podem manifestar-se no cenário da obra. Estruturas de concreto armado estão sujeitas à ação de carregamento e de esforços solicitantes, podendo ocasionar fissuras com aberturas que aproximam-se das provocadas nas amostras ensaiadas, como por exemplo na execução de reservatórios, tanques e demais obras expostas à água e pressão contínua. Sendo assim, é importante prever se o elemento estrutural onde será utilizado o concreto com aditivo cristalizante irá receber esforços que possam ocasionar a manifestação de fissuração com fissuras de aberturas superiores às indicadas pelo fabricante do aditivo, visto que, a sua incorporação nas amostras não apresentou resultados aparentes de autorregeneração ou de cristalização quando provocadas fissuras com aberturas maiores.

\subsection{Resistência à compressão axial}

A Tabela 9 apresenta os resultados de cada uma das amostras quando ensaiadas à resistência à compressão, em diferentes idades, nas amostras com e sem as fissuras provocadas. Os resultados obtidos com os ensaios nas amostras onde foram provocadas fissuras previamente estão representados com a letra ' $F$ '" em sua nomenclatura nas tabelas de resultados expostas na sequência deste trabalho. 
Tabela 9-Resistência à compressão

\begin{tabular}{|c|c|c|c|c|c|}
\hline \multirow[b]{2}{*}{ Traço } & \multicolumn{5}{|c|}{ Resistência a compressão (Mpa) } \\
\hline & Amostra & 07 dias & 14 dias & 28 dias & 56 dias \\
\hline \multirow[b]{4}{*}{ II.0,45(1).CR } & 1 & 44.3 & 48.8 & 49.8 & 54.8 \\
\hline & 2 & 47.7 & 49.8 & 48.9 & 52.6 \\
\hline & $3-\mathrm{F}$ & 49.3 & 46.6 & 52.0 & 56.5 \\
\hline & $4-\mathrm{F}$ & - & - & 51.8 & 53.3 \\
\hline \multirow[b]{4}{*}{ II.0,45(2) } & 1 & 49.5 & 52.5 & 54.2 & 54.0 \\
\hline & 2 & 49.5 & 49.7 & 54.8 & 55.2 \\
\hline & $3-\mathrm{F}$ & 50.1 & 47.4 & 52.7 & 52.9 \\
\hline & $4-\mathrm{F}$ & - & - & 51.6 & 53.3 \\
\hline \multirow[b]{4}{*}{ II.0,70(3).CR } & 1 & 30.3 & 30.0 & 32.5 & 35.0 \\
\hline & 2 & 26.9 & 32.1 & 31.5 & 34.3 \\
\hline & $3-\mathrm{F}$ & 30.9 & 29.3 & 31.2 & 34.0 \\
\hline & $4-\mathrm{F}$ & - & - & 33.2 & 34.3 \\
\hline \multirow[b]{4}{*}{ II.0,70(4) } & 1 & 28.9 & 33.2 & 34.7 & 32.7 \\
\hline & 2 & 29.3 & 33.1 & 32.5 & 34.8 \\
\hline & $3-\mathrm{F}$ & 34.0 & 33.7 & 33.7 & 33.6 \\
\hline & $4-\mathrm{F}$ & - & - & 34.6 & 34.5 \\
\hline \multirow[b]{4}{*}{ IV.0,45(5).CR } & 1 & 30.0 & 35.8 & 48.8 & 52.8 \\
\hline & 2 & 28.2 & 36.6 & 49.9 & 53.3 \\
\hline & $3-\mathrm{F}$ & 30.9 & 34.9 & 49.7 & 52.1 \\
\hline & $4-\mathrm{F}$ & - & - & 48.4 & 50.3 \\
\hline \multirow[b]{4}{*}{ IV.0,45(6) } & 1 & 34.2 & 37.3 & 50.2 & 52.5 \\
\hline & 2 & 34.1 & 39.8 & 48.8 & 49.8 \\
\hline & $3-\mathrm{F}$ & 31.4 & 40.0 & 50.7 & 51.7 \\
\hline & $4-\mathrm{F}$ & - & - & 49.4 & 51.5 \\
\hline \multirow[b]{4}{*}{ IV.0,70(7).CR } & 1 & 13.7 & 21.3 & 26.0 & 25.5 \\
\hline & 2 & 13.1 & 19.1 & 24.4 & 27.6 \\
\hline & $3-\mathrm{F}$ & 14.5 & 19.7 & 24.8 & 25.5 \\
\hline & $4-\mathrm{F}$ & - & - & 26.1 & 26.6 \\
\hline \multirow[b]{4}{*}{ IV.0,70(8) } & 1 & 16.7 & 18.0 & 20.3 & 24.0 \\
\hline & 2 & 16.5 & 18.0 & 24.4 & 25.1 \\
\hline & $3-\mathrm{F}$ & 17.2 & 20.2 & 24.6 & 23.9 \\
\hline & $4-\mathrm{F}$ & - & - & 23.9 & 24.5 \\
\hline
\end{tabular}

Aos 7 dias de idade observa-se que as amostras com cimento do tipo CP II F 40, apresentaram um maior ganho de resistência quando comparados aos traços de igual composição com uso de cimento do tipo CP IV. As amostras que receberam a incorporação do aditivo apresentaram resultados inferiores na resistência do concreto à compressão axial aos 7 dias de idade, enquanto aos sem o aditivo, estes apresentaram resultados mais elevados. Para o traço 1 com relação a/c igual a 0,45 e cimento CP II com incorporação de aditivo cristalizante, houve um ganho de $15 \%$ de resistência aos 56 dias com relação à idade inicial, enquanto ao sem aditivo, o acréscimo foi de $10 \%$ em sua resistência. Para os traços com relação a/c 0,45 e cimento CP IV o ganho de resistência na idade final de ensaio quando comparada à idade aos 7 dias foi de $72 \%$ para a composição com aditivo e de 54\% para a sem o aditivo. O ganho de resistência nas amostras com aditivo foi mais lento nas primeiras idades, porém observou-se um melhor comportamento nas composições no decorrer do tempo, alcançando resistências mais elevadas nos traços com cimento CP II. Quando comparados os traços com as mesmas amostras sem incorporação do aditivo, estes apresentam resistências mais 
elevadas que os traços com cristalizante. Desta forma, observa-se que a incorporação do aditivo cristalizante promove uma alteração no comportamento do concreto, alcançando resistências à compressão axial inferiores em idades iniciais, contudo, as amostras apresentam ganhos de resistência maiores em idades mais elevadas, ou seja, desenvolvem-se ao longo das idades com maiores incrementos de resistência. Nos Gráficos 1 e 2 são apresentados os maiores resultados das amostras quando ensaiadas à compressão com a idade na qual o ensaio foi realizado.

Gráfico 1 - Resistência à compressão axial em função da idade do concreto para amostras com a/c 0,45

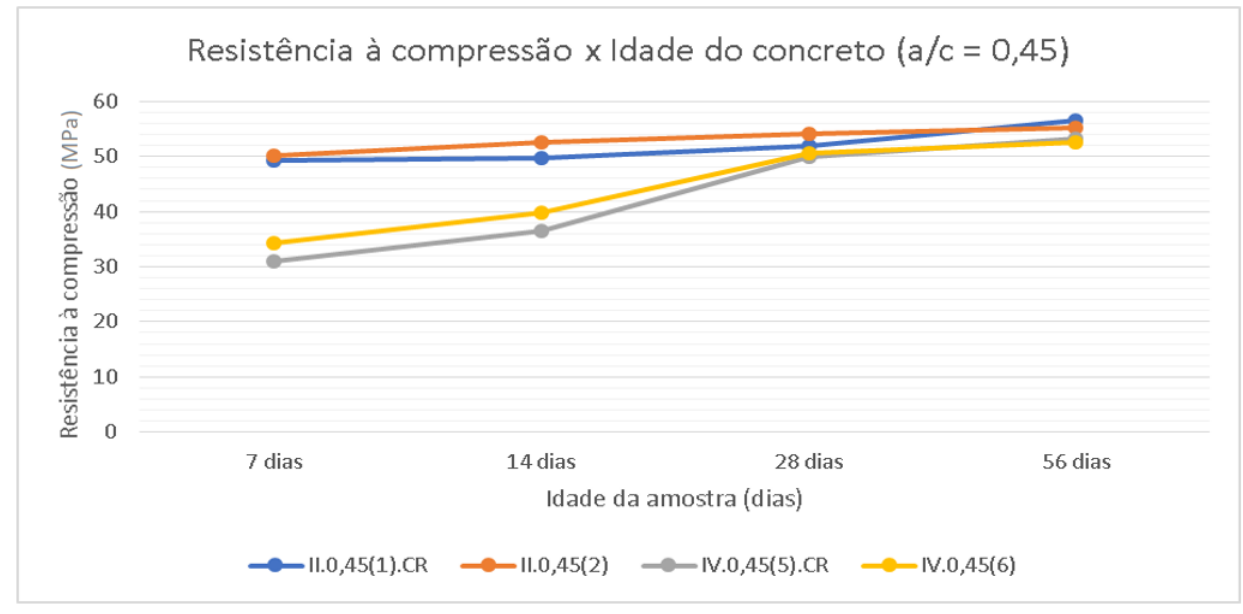

Gráfico 2 - Resistência à compressão axial em função da idade do concreto para amostras com a/c 0,70

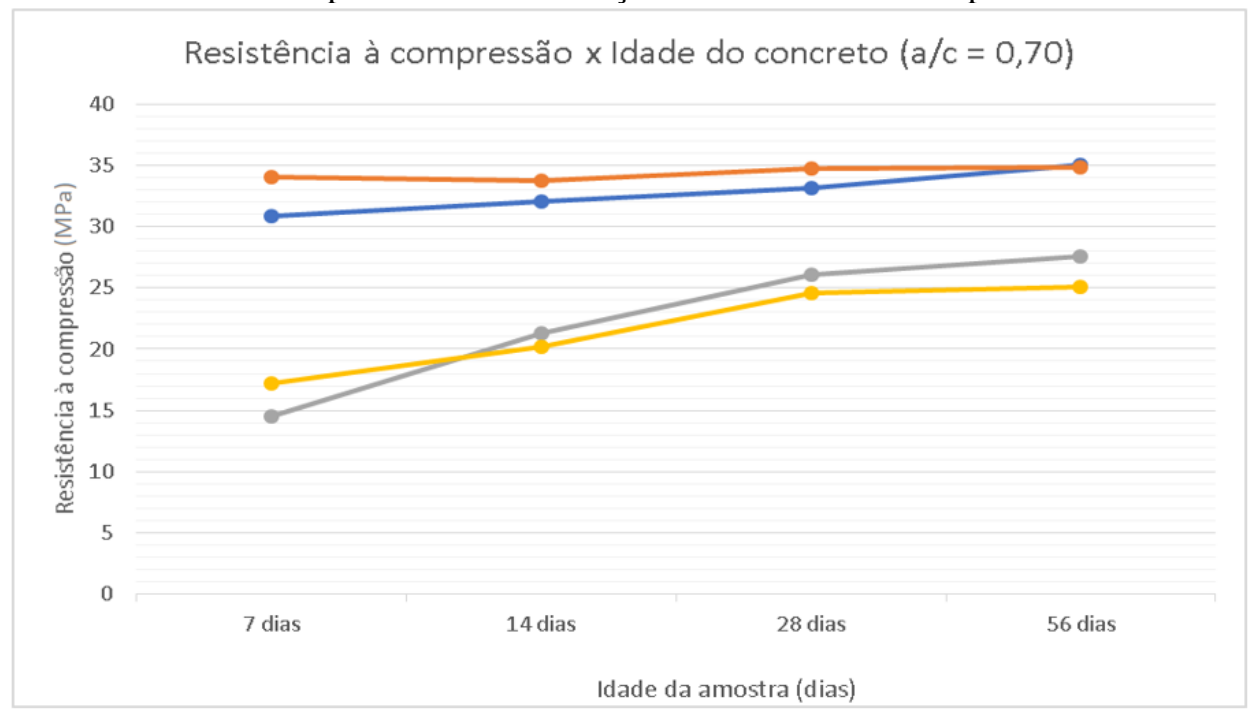

\section{CONCLUSÃO}

O programa experimental promoveu uma análise comparativa das propriedades mecânicas do concreto, sendo relacionados os traços convencionais aos de igual composição com a incorporação de aditivo cristalizante em suas misturas. Os resultados obtidos foram analisados e foi possível verificar que a incorporação do aditivo nas amostras influencia o comportamento e os parâmetros do concreto quando comparado às amostras sem adição do cristalizante, nos estados fresco e endurecido.

A resistência à compressão axial do concreto foi o principal parâmetro analisado neste trabalho, avaliada nas idades de 7, 14, 28 e 56 dias de idade. Observou-se que as amostras com a presença do aditivo em sua composição apresentaram um menor ganho de resistência nas idades iniciais quando comparadas aos traços referências sem incorporação do cristalizante. Sendo assim, a sua incorporação é válida quando almeja-se a atuação do agente cristalizante em estruturas ou situações específicas, tendo em vista que o início de sua contribuição como agente autorregenerante será efetivo após um tempo maior de cura, sendo possível identificar sua atuação a partir dos 28 dias de idade das amostras. As fissuras provocadas nas amostras possuíam aberturas maiores que as com eficácia de fechamento comprovadas pelo fabricante 
do aditivo, sendo executadas em maior dimensão para verificar a possibilidade de ser observado o fenômeno de autocicatrização e autorreneração do concreto. Contudo, com base nos resultados obtidos aos 56 dias de idade, verificou-se que as aberturas de fissuras não apresentaram uma redução ou completo fechamento. Portanto, esse trabalho reativa a ineficácia dos aditivos cristalizantes para a autorregeneração do concreto quando há o surgimento de fissuras com maiores dimensões.

\section{REFERÊNCIAS}

ASSOCIAÇÃO BRASILEIRA DE NORMAS TÉCNICAS. NBR 5739: Concreto - Ensaio de compressão de corpos de prova cilíndricos. Rio de Janeiro, 2018.

AHN, T. H.; KISHI, T. Crack self-healing behavior of cementitious composites incorporating various mineral admixtures. In: Journal of Advanced Concrete Technology 8, no. 2, p. 171-186. 2010.

Ferrara, L., Van Mullem, T., Alonso, M.C., Antonaci, P., Borg, R.P., Cuenca, E., ... De Belie, N. (2018). Experimental characterization of the self-healing capacity of cement based materials and its effects on the material performance: A state of the art report by COST Action SARCOS WG2. Construction and Building Materials, 167, $115-142$. https://doi.org/10.1016/j.conbuildmat.2018.01.143

MEDEIROS, M. H. F.; ANDRADE, J. J. O.; HELENE, P., A. F. Cimento Portland. In: Isaia G. C. (Ed) - Concreto: Ciência e Tecnologia. 1. ed. São Paulo, 2011. Cap. 22

LI, V. C.; HERBERT E. Robust Self-healing concrete for sustainable infrastructure. Journal of advanced concrete technology. Japan Concrete Institute, 2012.

MEHTA, P. K; MONTEIRO, P. J. M. Concreto: Microestrutura, Propriedades e Materiais $-1^{\mathrm{a}}$ ed. São Paulo: IBRACON, 2014.

MORI, S.; KURAMOTO, Y.; TAKAGI, E.M.; HORIE, M.; \& TANIMOTO, S. Repair of road bridge RC floor slab with advanced cracks from the floor underside. 51st Annual Meeting of the Civil Engineering Society of Japan, Vol 5, p. 344-345). Tokyo, 1996.

NEVILlE, A. M.; J. J. Tecnologia do concreto. 2.ed. Tradução Ruy Alberto Cremonini - Porto Alegre: Brookman, 2013.

OURIVES, C. N.; BILESKY, P. C. Avaliação do Desempenho dos Sistemas de Impermeabilização por Cristalização Capilar do Concreto. In: 50. Congresso Brasileiro do Concreto - Ibracon, Salvador, Brasil, 2008.

SISOMPHON, K.; COPUROGLU, O.; KOENDERS, E.A.B. Self-healing of surfasse cracks in mortars with expansive additive and crystalline additive. Cemente \& Concrete Composites, Vol 34, p. 566-574, 2012.

TAKAGI, E.M.; LIMA, M.G.; HELENE, P.R.L. Contribuição para estudo do efeito da autocicatrização em concretos ativado por catalisadores cristalinos em estruturas de túneis submetidas à exposição contínua de água. In: 3 . Congresso Brasileiro de Túneis e Estruturas Subterrâneas - SAT, São Paulo, 2012.

VAN BREUGEL, K. Is there a market for self-healing cement- based materials? In: Proceedings of the First International Conference on Self-healing materials, Noordwijk, Netherlands, p 1-9, 2007. 